\title{
Enfermedad de Alzhéimer e Implicaciones Diagnósticas Futuras
}

\author{
Gallego-Tavera, Sindy Yuliana ${ }^{1,2\left(^{*}\right)}$; Gómez-Montoya, María Isabel²; David Gonzales, Tatiana² \\ ${ }^{1}$ Vicerrectoría de Investigaciones, Grupo GORAS, Universidad Católica Luis Amigó, Facultad de psicología \\ Medellin, Colombia \\ ${ }^{2}$ Universidad de Medellín, Facultad de Derecho, Medellín, Colombia
}

\section{RESUMEN}

La enfermedad del alzhéimer (EA) es un tipo de demencia caracterizada por el deterioro cognitivo y neurofisiológico de tipo progresivo, de gran incidencia en la actualidad, debido a la cantidad de personas que la padecen a nivel mundial, la proyección exponencial, y los altos costos en tratamientos paleativos a los pacientes y la atención de enfermedades psicológicas a cuidadores por las secuelas causadas. Aunque en Colombia se presenta una variante de EA poco frecuente conocida como demencia frontotemporal en personas jóvenes, el foco patológico de la EA, son los adultos mayores, quien por lo genera presentan delicados cuadros clínicos concomitantes. Pese a que es una enfermedad conocida hace algún tiempo y sus avances referentes a la patología han aumentado, obteniendo biomarcadores, conociendo la importancia de alguna proteína y el factor genético han sido de gran ayuda en el desarrollo de la EA, a la actualidad se conocen pocos avances en la detección temprana, los tratamientos a esta y una posible cura, respecto a la velocidad con que avanza dicha enfermedad luego de la aparición de sus primeros síntomas.

Palabras clave: enfermedad de alzheimer; deterioro cognitivo; demencia; neurodegenerativo.

\section{Alzheimer's disease and future diagnostic implications.}

\section{ABSTRACT}

Alzheimer's disease (AD) is a type of dementia characterized by progressive cognitive and neurophysiological deterioration, whit a high incidence today, due to the number of people who suffer from it worldwide, the exponential projection, and the high cost in palliative treatments to patients and care of psychological illnesses to caregivers for the conse quences caused. Although in Colombia there is an infrequent variant of Ad known as frontotemporal dementia in Young people, the pathological focus of $A D$ is the elderly, who therefore present delicate concomitant clinical symptoms. Although it is a know disease some time ago and its advances regarding pathology have increased, obtaining biomarkers, knowing the importance of some proteins and the geenetic factor have been of great help in the development of $A D$, to date they are known few advances in early detection, treatments fot this and a posible cure, regarding the speed with which the disease progresses after the appearance of its firt symptoms.

Keywords: alzheimer's disease; cognitive; impairment; dementia; neurodegenerative. 


\section{INTRODUCCIÓN}

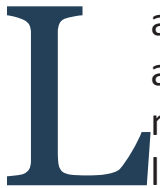

a Enfermedad de Alzhéimer (de ahora en adelante, EA) es una de las causas de demencias más reconocidas en el mundo por lo cual la Organización Mundial de la Salud - OMS, considera la EA como prioridad mundial de salud pública en términos epidemiológicos, debido al crecimiento exponencial de esta en el mundo. Aun cuando se han generado avances en la comprensión, patología y conceptualización de la EA, desde que Alois Alzhéimer reportó el primer caso, no se han encontrado tratamientos reveladores, dando continuidad a la preocupante realidad de que actualmente ninguna enfermedad neurodegenerativa tiene cura (Zhang, Gu, Shen, Liu y Lin, 2017).

Existen pruebas de que las muertes por EA han incrementado en las últimas décadas pese a los tratamientos paliativos, siendo la única que además de no tener cura, no permite postergar sus efectos. La demencia, es denominada crónica, ya que en términos económicos los gastos a nivel global rondan los $\$ 604,000$ millones de dólares americanos - USD, y solo en gastos el abordaje paliativo de la EA se calcula que del 2000 al 2015 a la Sociedad Estadounidense le costó cerca de \$ 226 millones de USD. En el año 2014 se evaluó que la EA no solo afecta a quien la padece, sino también a sus familiares, cuidadores y círculos sociales, ocasionando en ellos, variedad de enfermedades psicológicas; debido a las 17,9 mil millones de horas de cuidado no remunerado a la atención primaria de estas personas, motivo por el cual, se incrementara el número de consultas médicas de cuidadores costando en el mismo año alrededor de $\$$ 9,7 mil millones de USD, por ese motivo se requiere el aumento de la inversión otorgada a centros especializados en investigación básica para la búsqueda de mecanismos eficaces en la intervención y cura de la EA, además de nuevos paradigmas transdisciplinarios para minimizar los síntomas y mejorar la calidad de vida de los pacientes, familias y cuidadores. (De-La-Peña, Parra-Bolaños y Fernández-Medina, 2018; Ruiz-Vargas, Sposato, Lee, Hachinski y Cipriano, 2018).

En la etapa senectud, los humanos experimentan un deterioro conocido como proteostasis, que es un fenómeno neuroquímico y proteico mediante el cual las proteínas del cerebro se convierten en agentes estabilizadores del sistema nervioso central - SNC. Las alteraciones en la proteostasis conducen a un elevado grado de acumulación de proteínas de características neurofibrilares que afecta los cuerpos de
Lewy y los procesos de mielinización del encéfalo. Los daños también se detectan a nivel neuroanatómico en las placas extracelulares, produciendo una activación masiva de las placas seniles y de priones, permitiendo diagnosticar la EA a partir de dos patologías fundamentales y únicas: el daño en las placas seniles y en los ovillos neurofibrilares (Lu, Harris, Shiroma, Leung y Kwok, 2018; Wang, Zhang, Lei y Zhao, 2018).

\section{MARCO TEÓRICO}

\section{Epidemiología}

La demencia conocida como EA, causa deterioro cognitivo y neurofisiológico de tipo progresivo, motivando así mismo el deterioro en las actividades cotidiana de los sujetos. Las investigaciones recientes sugieren que 44 millones de personas viven con demencia en todo el mundo en la actualidad. Según registros de los Institutos Nacionales de la Salud desde 2015, solo en Estados Unidos 5, 3 millones de estadounidenses que padecen EA, alrededor de 5, 1 millones son adultos mayores; con una proyección para el 2050 de 13,8 millones de personas aproximadamente, con un costo anual de la atención requerida a los pacientes, podría exceder fácilmente los 600,000 millones de USD (Albrecht, Hanna, Kim y Perfetto, 2018).

En Inglaterra y Gales, la demencia es la principal causa de muerte, evidenciando un $11,6 \%$ de todas las defunciones registradas en 2015 se correspondan con la EA. Por otra parte, se han hallado estudios recientes en los que se sugiere que la incidencia de la demencia, especialmente en los hombres, podría estar disminuyendo en los países occidentales, aunque aún no se sabe con certeza cuáles son las causas, esto podría servir como biomarcador para mejorar el manejo del riesgo neurovascular sufrido por pacientes con demencias. La EA es la culpable de la mayor cantidad de casos reportados de demencia, dado que representa entre el 50\% - 75\% de las mismas (Ishikawa, Yoshitomi, Covey, Zorumski y Izumi, 2018).

\section{Etiología de la enfermedad}

La EA se presenta en la vida de los sujetos frecuentemente de una manera esporádica, en la cual se expresan genes, tales como la presenilina 1 y 2 , y la 
proteína precursora de amiloide. Normalmente los síntomas se evidencian entre los 50 y 65 años de edad, así mismo es posible que la EA de inicio tardío exhiba una relación entre factores ambientales y genéticos, con el $70 \%$ del riesgo de adquirir la EA debido al factor genético, donde el gen APOE con sus tres variantes e2, e3 y e4 se convierte en el más peligroso para la aparición de la EA esporádica en equiparación con las portadoras no-e4 y e4, los heterocigotos tienen un Radio de Odds ratio - OR para la EA de 3 puntos, elevándose a 12 puntos en casos de homocigotos, lo que es un biomarcador que complica aún más el abordaje clínico de la EA (Umar y Hoda, 2017).

Los estudios epidemiológicos recomiendan que, para ralentizar los síntomas de aparición de la EA, se deben realizar actividades tales Como ejercicio físico y mental. Es necesario generar más estudios experimentales para contrarrestar la aparición temprana de la EA, así como un abordaje multidisciplinario de esta demencia, dado que los pacientes también suelen presentar cuadros clínicos de hipertensión y diabetes, perjudicando la calidad de vida de poblaciones con EA. La influencia de la EA hasta la actualidad continua sin estar clara, sin embargo, sigue siendo foco de estudio, ya que existen pocos datos epidemiológicos que provean información decisiva sobre cómo contrarrestar esta patología (Parra-Bolaños, Fernández-Medina y Martínez-Restrepo, 2014).

Las principales características cardinales de la patología de la EA son los daños en las placas amiloides y en componentes neurofibrilares, como ya se mencionó, de igual forma se encuentran también neuritas distróficas con problemas de activación microglial, daños en los hilos del neuropilo y angiopatía. Esto trae consigo graves consecuencias como la neurodegeneración del sistema nervioso simpático y parasimpático, además de daños sináptico y pérdida neuronal que conduce a atrofia macroscópica, sin dejar de lado que en edades muy avanzadas se presentan con la EA cuadros clínicos concomitantes sumamente delicados Dai, Zheng, Zeng y Zhang, 2017; Lehrer, Rheinstein y Rosenzweig, 2018).

Es menester señalar que los ovillos neurofibrilares generalmente están compuestos de filamentos helicoidales apareados, los cuales se fundamentan en la proteína tau hiperfosforilada, que habitualmente inicia sus movimientos por regiones del lóbulo temporal medial, abarcando la corteza entorrinal y el hipocampo, mucho antes de propagarse a zonas del isocortex, presentando daños severos en las áreas visuales primarias, además de la masa encefálica de- bido a la pérdida significativa de tamaño y peso, a causa de la formación de placas de proteína tau, y por ende los criterios clínicos y la gravedad de la EA están estrechamente correlacionadas con la presencia de beta-amiloide la cual es un biomacador, quizá el más importante, para la aparición de la EA (Pchitskaya, Zhemkov y Bezprozvanny, 2018).

\section{Patogénesis}

La teoría más significativa de la EA, se basa en la hipótesis amiloide, debido a la acumulación de formas patológicas de la proteína beta-amiloide creada por la escisión secuencial de la aplicación de las enzimas b y c secretadas al cerebro siendo este el primero proceso patológico, promovido por la inestabilidad que se presenta en $\mathrm{Ab}$ y $\mathrm{Ab}$ de despeje. Como es sabido, la importancia de Ab proviene esencialmente de la genética, lo que conlleva que la EA tenga mutaciones que están interconectadas en el procesamiento de $\mathrm{Ab}$, dando una sobreproducción relativa de sustancias toxicas de beta-amiloide (Jojo, Kuppusamy, De y Karri, 2019).

En la actualidad se piensa que el causante de la EA proviene originalmente de los oligómeros de $A b$ solubles, en donde los oligómeros purificados de cerebros con EA, producen inhibición de la potencialización a largo plazo de los sistemas sinápticos, generando muerte cerebral en los pacientes con EA. En algunos casos, los oligómeros también incitan procesos de hiperfosforilación de la proteína tau en epítopos significativos para la aparición de la EA, causando distrofia neurítica en neuronas sanas. Por otro lado, las placas cumplen un papel fundamental, pues estas pueden actuar como un depósito desde el cual los oligómeros amiloides se difunden, de igual modo pueden cumplir la función de mecanismo protector de secuestro tóxico de la serie Ab hasta alcanzar el punto de saturación fisiológica (Ohsawa, Tanaka, Ehara, Makita y Onaka, 2017).

Se evidencia que tanto para la patología Ab como para la tau se presentan falencias, debido a que las mutaciones en el gen de tau conducen a la acumulación de la misma y a la presencia de una diversidad de demencias neurodegenerativas dentro del espectro de demencias frontotemporales. Debido a que las mutaciones tau no conducen a la causa definitiva de adquirir la EA, se requieren estudios que permitan identificar las debilidades de la EA en animales no humanos, de modo que dichos hallazgos generen nuevos enfoques hacía la enfermedad (Suzuki, Iwata 
y Iwatsubo, 2017).

En la actualidad se han revelado exitosos estudios, los cuales dan a conocer que la aparición de los biomarcadores de líquido cefalorraquídeo - LCR y el uso de tomografía por emisión de positrones (por sus siglas en inglés, PET), demuestran a nivel clínico que la proteína beta-amiloide y la patología tau, tienen un desarrollo progresivo muy similar, lo que arroja pistas sobre las dinámicas de estos componentes en el SNC de los pacientes. Dichos estudios fueron realizados tanto en pacientes ancianos con EA esporádica y adultos mayores sanos. Se obtuvo que la patología amiloide se desarrolla mucho tiempo antes de que los síntomas clínicos puedan verse reflejados en un paciente y estos se anticipan a los cambios en la proteína tau. La EA tiende a progresar con relación a la patología tau sin beta-amiloide, lo cual es posible que sea parte del proceso normal del envejecimiento, haciendo necesario ejecutar estudios revolucionarios en los que se pueda determinar la correlación de dependencia entre las enfermedades neurodegenerativas y la senectud (Ismaili y Do-Carmo-Carreiras, 2017).

Algunas de las proteínas de la EA se dirigen a ciertas regiones del cerebro y las afectan, mientras que otras regiones no se ven dañadas por estas, haciendo posible que se demostrara que cuando $A b$ y tau están anormalmente plegados, pueden inducir a cambios conformacionales en la estructura normal de los neuropéptidos, así mismo como ocurre con la enfermedad priónica. El lugar de la patología original podría posiblemente determinar qué redes corticales se pueden ver afectadas y por medio de la ruptura de las redes diferenciales se nos permite interpretar la diversidad fenotípica observada en la EA (Romero y Marco-Contelles, 2017).

En la patogenia de la EA, se ha visto una crítica hacia la patología amiloide y tau desde otros enfoques clínicos, aunque no se ha precisado cómo están vinculadas mecánicamente las dos, se han proporcionado datos, que proponen que el sistema inmune innato juega un papel crítico en la patogénesis de la EA y es el responsable de generar este enlace. Se evidencia por otro lado una cantidad de genes de riesgo de la EA incluyendo CR1, CD33 y TREM2, los cuales están conectados con las vías del sistema inmunitario (Lu, Zhao, Yao, Xu y Ma, 2018).
Regularmente, los sujetos diagnosticados con EA son en gran parte ancianos, los que presentan signos de rápida progresión en cuanto a alteraciones en la memoria episódica. Se presentan de igual forma dificultades topográficas, dificultades con la multitarea y la pérdida de confianza hacia las personas que los rodean, así mismo se van presentando diversas dificultades cognitivas, alterando tanto en el desarrollo de su vida cotidiana, como en la anatomía y fisiología de sus cerebros. Justo en esta etapa se puede pronosticar la EA, pero dicho diagnóstico, sigue teniendo el grave defecto de ser sumamente tardío. La regla general es que la enfermedad se va desarrollando junto a diferentes patologías, entre las que se encuentran la perdida de movilidad, alucinaciones y en determinados casos, convulsiones. La muerte puede suceder en aproximadamente ocho años y medio después de los primeros síntomas (Mazumder y Choudhury, 2019).

Se han hallado síndromes poco típicos que padecen mayormente las poblaciones jóvenes, en ellos pueden presentarse síntomas como atrofia cortical posterior, afasia logopénica y la variante frontal de la EA, la cual puede confundirse con la demencia frontotemporal, ya que esta primera suele ser muy inusual, presentándose en edades comprendidas entre los 25 a 30 años para el particular caso de poblaciones colombianas. Existen mutaciones que están ligadas con unas características especiales como convulsiones y paraparesia espástica (Cheng, Yang y Zhang, 2019; Guzmán-Vélez y Cols, 2018).

\section{Criterios diagnósticos}

Teniendo como base fundamental que las patologías empezar a desarrollarse verse años antes de que se presenten los primeros síntomas, los factores de diagnóstico han sido modificados para que el diagnóstico diferencial se pueda realizar cada vez más temprano y con un alto énfasis en los componentes moleculares de la enfermedad, lo que es un factor muy positivo respecto de la evolución en las tecnologías de detección precoz de la EA. Recientes criterios diagnósticos elaborados por el Instituto Nacional de Envejecimiento y el Grupo de Trabajo Internacional para la EA, han podido sumar muchas fases preclínicas para la comprensión de la EA. Para poder diagnosticar la EA es necesario contar con rasgos propios de patologías que confirmen los criterios que llevan a la demencia o el deterioro cognitivo leve conducente 
a la EA. También existe la probabilidad de reconocer situaciones poco usuales de tipo no amnésicas (Morello, Lima y Brandão, 2017).

Los pacientes pueden acudir frecuentemente a servicios de neurología por pérdida de memoria y el diagnóstico puede realizarse por múltiples causas hasta localizar las principales variables que inciden en la aparición de la EA. Sumado a ello, también se reportan casos de personas con ansiedad severa asociada a pérdida de memoria, trastornos psicológicos y consumo de sustancias psicoactivas, lo que quiere decir que es necesario hacer diagnósticos duales para identificar claramente la EA en correlación con otros cuadros clínicos. Existen otras formas de la EA, las que pueden incluir la demencia por Cuerpos de Lewy y la demencia frontotemporal, presentándose mediante deterioro cognitivo vascular, infeccioso o inflamatorio. Se evidencian también una serie de causas que van desde la amnesia epileptiforme transitoria hasta la apnea del sueño (Gul, Bakht y Mehmood, 2019).

Para el adecuado diagnóstico de la EA, se siguen dos pasos fundamentales como lo son: la evaluación clínica y la entrevista clínica con el paciente. Este examen debe hacerse con un acompañante $y$ preferiblemente conexo a un examen físico focalizado. La neuropsicología permite que se puedan identificar plenamente los déficits cognitivos relacionadas con la edad. Se deben realizar exámenes rutinarios de sangre que permitan excluir ciertas características que puedan inducir a los síntomas cognitivos, los cuales normalmente incluyen hemograma, función tiroidea, ácido fólico, vitamina B12 y función renal. A criterio de los especialistas y tomando como base el entorno clínico, se puede tomar la decisión de excluir un rango de causas inflamatorias, metabólicas e infecciosas por medio de pruebas serológicas específicas (Kang, Park, Kim y Yi, 2018).

Una imagen estructural por medio de una tomografía axial computarizada - TAC, es recomendable en los pacientes que están en proceso de diagnóstico por deterioro cognitivo, esto con el fin de excluir posibles anomalías y poder hacer un diagnóstico más positivo y detallado. Cuando se presenta atrofia temporal y se evidencian daños a nivel parieto-occipital se presenta atrofia con poca conservación del hipocampo, esto cuando la enfermedad está en sus primeras fases. Se pueden excluir por medio de la Resonancia Magnética Funcional - RMF otras enfermedades neurodegenerativas, así como una posible extensión de enfermedades cerebrovasculares, por medio de dicha técnica (Sun, Zhang y Chen, 2017).

Actualmente disponemos de una serie de técni- cas de neuroimagen como la tomografía por emisión de positrones - PET para identificar los efectos de los amiloides sobre diversas regiones encefálicas. Recientemente el PET está evaluando los efectos del beta-amiloide en la mayoría de casos de EA que se rastrean prácticamente a nivel global, por medio de recursos que permiten revelar su utilización clíni$\mathrm{ca}$, aunque para las naciones con mayores déficits económicos y financieros, es comprensible un escaso uso del PET para trabajos clínicos, lo que debería modificarse mediante planes de adquisición de equipos financiados para mejorar el acceso a tecnologías de neuroimagen en pro de diagnosticar con mayor eficacia a poblaciones con EA de naciones en vías de desarrollo y no sólo en ciencias básicas (Morley, Farr y Nguyen, 2018).

Un examen de líquido cefalorraquídeo - LCR, puede conducir a los expertos a descartar posibles causas poco comunes y un posible cuadro clínico de EA. Este examen sirve como herramienta diagnostica molecular positiva en poblaciones ancianas. Existe un patrón muy típico de LCR en EA, siendo este un poco bajo en cantidades de Ab42 y unos niveles altos en tau y en fosfo-tau. El anterior patrón es de vital importancia para la predicción de aparición de la EA, por lo que es una herramienta indispensable para diagnósticos de alta precisión. Existe una técnica que mediante la utilización de paneles genéticos y utilizando secuenciación de última generación, permite la producción masiva de genes, que sirven como prueba de coste razonable y que resulta más accesible para centros especializados con escasos recursos (Paumier y Thinakaran, 2019).

\section{Tratamiento y manejo}

Una gestión optimizada de tratamientos paliativos debe ser empleada según el paciente y su circunstancia, lo cual permitirá adaptar la calidad de vida del mismo a las condiciones que poco a poco va imponiendo la enfermedad. Los tratamientos preventivos le permiten al paciente presentar leves progresos cognitivos y funcionales, haciendo que, si el proceso involucra a la neuropsicología clínica, se pueda fomentar que los cuidadores sean parte central del proceso y más aún, en la toma de decisiones. El paciente por su parte debe estar consciente de todos los pasos de su evolución, inclusive cuando el desarrollo cognitivo parezca disminuir; por lo que se debe abordar la EA desde enfoques multidisciplinarios. Es necesario que los pacientes puedan contar con un apoyo emo- 
cional permanente, con respaldo financiero y con una clara estructuración del futuro, en tanto que el paciente vaya adquiriendo las facultades que le permitan tomar decisiones independientemente de sus cuidadores y teniendo clara y lúcida su percepción de calidad de vida. Solo la decisión de darle al paciente cuidados paliativos para la planificación de la etapa final de su vida, es una determinación que debe ser tomada preferiblemente antes de la demencia terminal (Belloy, Napolioni y Greicius, 2019).

Los inhibidores de la acetilcolinesterasa, son fundamentales para un tratamiento sistemático, pueden presentarse efectos secundarios como: calambres en las extremidades inferiores, además de problemas gastrointestinales y a su vez estos pueden presentarse altos niveles de estrés y ansiedad. Deben evitarse o usarse con precaución, medicamentos cardiacos en individuos con EA de edades avanzadas, porque pueden causar bradicardias y arritmias. En famoso estudio denominado como DOMINO-AD, demostró que al retirar el donepezil en proporciones de moderadas a altas, provoca que pacientes diagnosticados con EA recurran un poco más tarde a hogares especializados, demostrando que se puede ralentizar la EA hasta en 12 meses de menos tratamiento, pero no en los siguientes 3 años, lo que hace que pese al grado de éxito de DOMINO-AD, siga siendo absolutamente insuficiente para detener la enfermedad, lo que quiere decir que se requieren nuevos modelos clínicos y experimentales para curar la EA (Ingale y Kasture, 2017).

La memantina es un antagonista del receptor $\mathrm{N}$-metil-D-aspartamo de poca afinidad, es un tratamiento sintomático alternativo, su finalidad es poder reducir la neurotoxicidad excitatoria del L-glutamato. Lo anterior sin obstaculizar con sus acciones fisiológicas, se pueden presentar algunos efectos como estreñimiento y dolor de cabeza. La memantina ha manifestado tener un beneficio insignificante, pero clínicamente apreciable en el deterioro funcional en personas con EA leve a grave. Existe evidencia de que la combinación de la utilización de IECA y memantina, sugiere mejores resultados. Un meta-análisis reciente encontró evidencia débil de mejoría de los síntomas de conducta en la EA moderada a grave con dicho tratamiento. Los trastornos como la depresión y la ansiedad son usualmente vistos en EA y tiene un impacto negativo sumamente significativo en la calidad de vida de los pacientes, presentando alteraciones del estado de ánimo en los cuidadores y un alto riesgo que los pacientes deban ser recluidos en centros especializados. Los fármacos antidepresivos pueden empeorar el problema y deben evitarse a toda costa, ya que no tienen efectos positivos en pacientes con EA (Kim, Jung y Yoon, 2018).

En las etapas posteriores de la demencia se puede desarrollar agitación, agresión y psicosis, para esto, la utilización de fármacos y sus resultados son más bien moderados y en sí, no hay tratamientos que estén garantizados cuando se está en una etapa de demencia avanzada. Cuando se está en etapas críticas se suelen presentar infecciones torácicas, apoplejías y de forma indefectible, la muerte. En otras palabras, los fármacos deben ser eludidos y solo usarse en lo posible, para mitigar algunos síntomas neuropsiquiátricos, particularmente psicosis, las que suelen agravarse al combinarse con estos fármacos y pueden causar síntomas debilitantes o presentar riesgos para la seguridad del paciente. Se sugiere que los tratamientos en estos casos se hagan desde un enfoque no-farmacológico que incluya entrenamiento en habilidades de comunicación, así como el entrenamiento en atención centrada en la persona y en los cuidadores (Doggrell, 2018).

\section{METOdOLOGÍA}

El desarrollo de esta investigación está basado en la reseña general de elementos que han marcado en la globalización. La búsqueda, selección, organización y análisis del material bibliográfico empleado en este artículo se sustenta en el tipo de investigación documental y se apoya en el enfoque cualitativo. En el material bibliográfico recopilado se emplearon un total de 90 textos producto de una exhaustiva búsqueda en bases de datos académicas tales como: JCR (Journal Citation Reports), ISOC, Iresie, Google Académico, Redalyc, Scielo, DICE (Difusión y Calidad Editorial de las Revistas Españolas de Humanidades y Ciencias Sociales y Jurídicas), EBSCO y Scopus. Los criterios básicos tenidos en cuenta en la selección de los documentos fueron: referentes normativos, políticos y programáticos adoptados por Colombia; hallazgos de investigaciones en el ámbito internacional y nacional; periodo de la publicación (1972-2019).

Para la estructuración y exploración del material, se hizo un listado de categorías donde se remitieron datos como: autor, año, referencia bibliográfica y país de publicación, conceptos básicos (globalización, migración, teléfono móvil, redes sociales, empleo, salario,...), características de las herramientas implementadas en el marco de las políticas de bilingüismo 
en Colombia; elementos relacionados con los aciertos, desaciertos, obstáculos y especificidades del contextos en el que se aplican dichas políticas. Como estrategia de trabajo se sirvieron de bases propias de un análisis documental el cual según Van Dijk (1995), consiste en implementar estructuras discursivas y significados, así como algunos aspectos u opiniones generales, teniendo en cuenta elementos psicológicos, cognitivos, contextuales e ideológicos, de ahí su complejidad interdisciplinaria.

\section{DISCUSIÓN}

En cuanto al aspecto psicosocial, es necesario que se haga uso de la neuropsicología clínica para la generación de modelos integrales entre los ámbitos afectivos, sociales, interpersonales, laborales, emocionales y de redes de apoyo que tengan los pacientes con EA, además de la generación de pruebas estandarizadas que puedan detectar tempranamente los primeros signos de presencia de EA, por lo que sería un desafío sumamente interesante, aplicar pruebas a toda la población adulta mediante sistemas similares a los de los censos poblacionales, para así poder regular y controlar los cuadros clínicos de pacientes con EA, para con base en dicha información, hacer un rastreo de las condiciones sociales que ralentizan o aceleran la pérdida de calidad de vida en personas con EA (Atri, 2019).

\section{CONCLUSIONES}

A pesar de que en los últimos años no se ha podido comprender muy bien la etiología de la EA, el estudio de la misma, ha tenido un progreso significativo en los últimos cinco años, aunque de igual forma queda mucho por hacer, ya que como es bien sabido, las enfermedades neurodegenerativas, hasta la fecha, no tienen cura, sino que solo hay tratamientos paliativos. Es por esto que se sugiere que, en los próximos estudios genéticos a realizar, se procure la generación de nuevos modelos más allá de los modelos estándar existentes, para conseguir innovadoras visiones y nuevos abordajes neurobiológicos y neurocientíficos sobre los orígenes y mantenimiento de la EA (Chakraborty, 2017; Orf y Walker, 2017; Parady, 2018).

El fracaso de los ensayos clínicos con anticuerpos dirigidos al cerebro ha hecho que el beta-amiloide genere escepticismo acerca de la hipótesis de la misma como la única causante de la EA, pero aun así es más preocupante que ciertas perspectivas de modificación de la EA, han tenido complicaciones debido a los objetivos y selección de pacientes, por lo que en este tipo de estudios se han encontrado tanto pacientes sanos como pacientes con EA, en los cuales el beta-amiloide ya no se cuenta como objetivo apropiado para definir la etiología de la EA (Kim, 2018).

Existen millares de ensayos clínicos con validez respecto de la relevancia de la proteína beta-amiloide como principal causante de la EA, los cuales denotan información importante sobre los resultados prometedores que están obteniendo los ensayos con roedores, sin embargo, aún faltan varias décadas de estudios y de análisis en comités de bioética y en tribunales estatales, antes de pasar a ensayos con muestras humanas. En cuanto a las terapias modificadoras de la EA, se está a la espera de que estas arrojen resultados positivos en los pacientes que contengan la EA, por lo que, un paso fundamental, consistirá en cerciorarse de que esto sea factible y que sea fácil de implementarse. Se debe estudiar siempre, cuáles son los sujetos que son más propensos a padecer de la enfermedad, con el fin de actuar a tiempo y poder identificar cuáles serán los nuevos biomarcadores y con base en ello, elaborar ensayos clínicos personalizados y comparándolos con sujetos que presenten signos de retraso en cuanto a la aparición de la EA (Das y Yan, 2019). 


\section{REFERENCIAS}

Acar, B. A. (2018). Impact of emergency room meetings on improvement of door-to-needle times in acute ischemic stroke patients: A single center's experience. Northern Clinics of Istanbul, 5(4):282-287. http://doi.org/ 10.14744/nci.2017.00378.

Bravo, G., Richards, C. L., Corriveau, H., \& Trottier, L. (2018). Converting Functional Autonomy Measurement System Scores of Patients Post-Stroke to FIM Scores. Physiotherapy Canada, 70(4):349355. https//doi.org/ 10.3138/ptc.2017-82.

Bright C. J., \& Cols. (2017). Risk of Cerebrovascular Events in 178962 Five-Year Survivors of Cancer Diagnosed at 15 to 39 Years of Age: The TYACSS (Teenage and Young Adult Cancer Survivor Study). Circulation, 135(13):11941210.https://doi.org/10.1161/ CIRCULATIONAHA.116.025778.

Bosiers M. J., \& Cols. (2018). Incidence and prognostic factors related to major adverse cerebrovascular events in patients with complex aortic diseases treated by the chimney technique. Journal of Vascular Surgery, 67(5):13721379.https://doi.org/10.1016/j.jvs.2017.08.079.

Burgos, E., Bermúdez, J., \& Chávez, W. (2018). Atrial myxoma related with ischemic cerebrovascular event. Medicina Clinica (Barcelona), 151(12):e69-e70. https//doi.org/10.1016/j.medcli.2018.04.004.

Chatterjee, A., \& Hillegass, W. B. (2018). Patent foramen ovale closure for cryptogenic stroke: Effective, but only in select patients. Catheterization and Cardiovascular Interventions, 92(1):174-175. https:// doi.org/10.1002/ccd.27700.

Chimatiro, G. L., Rhoda, A. J., \& De Wit, L. (2018). Stroke patients' outcomes and satisfaction with care at discharge from four referral hospitals in Malawi: A cross-sectional descriptive study in limited resource. Malawi Medical Journal, 30(3):152-158. https://doi.org/ 10.4314/mmj.v30i3.4.

De-La-Peña, C., Parra-Bolaños, N., \& Fernández-Medina, J. M. (2018). Análisis de la alfabetización inicial en función del tipo de familia. Ocnos, 17(1), 7-20. https://doi.org/10.18239/ocnos_2018.17.1.1336.

Erskine N., \& Cols. (2017). A systematic review and meta-analysis on herpes zoster and the risk of cardiac and cerebrovascular events. PLoS One, 12(7):e0181565. https:// doi.org/ 10.1371/journal.pone.0181565.

Guzmán-Vélez, E., \& Cols. (2018). A Three-Factor Structure of Cognitive Functioning Among Unimpaired Carriers and Non-Carriers of Autosomal-Dominant Alzheimer's Disease. Journal of Alzheimer's Disease: JAD, 65(1):107-115. https://doi.org/
He, W., Wang, Q., Gu, L., Zhong, L., \& Liu, D. (2018). NOX4 rs11018628 polymorphism associates with a decreased risk and better short-term recovery of ischemic stroke. Experimental and Therapeutic Medicine, 16(6):5258-5264. https://doi.org/ 10.3892/etm.2018.6874.

Hepburn, M., Bollu, P. C., French, B., \& Sahota, P. (2018). Sleep Medicine: Stroke and Sleep. Missouri Medicine, 115(6):527-532.

Hiragami, S., Nagahata, T., Koike, Y., \& Inoue, Y. (2018). Lower garment-lifting postural control characteristics during toilet-related activities in healthy individuals and a post-stroke hemiplegic patient undergoing rehabilitation. Journal of Physical Therapy Science, 30(12):1462-1467. https:// doi. org 10.1589/jpts.30.1462.

Hosseini, Z. S., Peyrovi, H., \& Gohari, M. (2019). The Effect of Early Passive Range of Motion Exercise on Motor Function of People with Stroke: a Randomized Controlled Trial. Journal of Caring Sciences, 8(1):39-44. https:// doi.org/ 10.15171/ jcs.2019.006.

Ghannam, A. S. B., \& Subramanian, P. S. (2017). Neuro-ophthalmic manifestations of cerebrovascular accidents. Current Opinion in Ophthalmology, 28(6):564-572.https://doi.org/10.1097/ICU. 0000000000000414.

Geltser, B., Kurpatov, I. G., Kotelnikov, V. N., \& Zayats, Y. V. (2018). Chronic obstructive pulmonary disease and cerebrovascular diseases: functional and clinical aspect of comorbidity. Terapevticheskii Arkhiv, 90(3):8188.https://doi.org/10.26442/terarkh201890381-88.

Glickman, L. B., \& Chimatiro, G. (2018). Clients with stroke and non-stroke and their guardians' views on community reintegration status after in-patient rehabilitation. Malawi Medical Journal, 30(3):174179. doi: 10.4314/mmj.v30i3.8.

Güner, A. (2018). Left Atrial Thrombus Presenting with Acute Coronary Syndrome and Cerebrovascular Event. The Journal of Tehran University Heart Center, 13(2):99-100.

Katzan, I. L., Schuster, A., Newey, C., Uchino, K., \& Lapin, B. (2018). Patient-reported outcomes across cerebrovascular event types: More similar than different. Neurology, 91(23):e2182-e2191. https://doi. org/ d10.1212/WNL.0000000000006626.

Kim, A., Hong, S. B., Lee, S., Mo, H., \& Koo, D. L. (2018). Acute Ischemic Stroke Mimicking Non-Convulsive Status Epilepticus. Journal of Epilepsy Research, 8(2):90-92. https//doi.org/ 10.14581/jer.18015.

Kim J. C., \& Lim, J. H. (2018). The effects of coordinati- 
ve locomotor training on coordination and gait in chronic stroke patients: a randomized controlled pilot trial. Journal of Exercise Rehabilitation, 14(6):1010-1016. https:// doi.org/ 10.12965/ jer.1836386.193.

Manners, J., Steinberg, A., \& Shutter, L. (2017). Early management of acute cerebrovascular accident. Current Opinion in Critical Care, 23(6):556-560. https:/7doi.org/10.1097/MCC.0000000000000462.

Lewek, M. D., Raiti, C., \& Doty, A. (2018). The Presence of a Paretic Propulsion Reserve During Gait in Individuals Following Stroke. Neurorehabilitation and Neural Repair, 32(12):1011-1019. https:// doi. org/ 10.1177/1545968318809920.

Lin, D. J., Finklestein, S. P., \& Cramer, S. C. (2018). New Directions in Treatments Targeting Stroke Recovery. Stroke, 49(12):3107-3114. https://doi. org/10.1161/STROKEAHA.118.021359.

Lindow, T., \& Baranchuk, A. (2018). Interatrial block and ischemic stroke. Journal of Thoracic Disease, 10(12):7052-7054. https://doi.org/ 10.21037/ jtd.2018.11.23.

Parra-Bolaños, N., Fernández-Medina, J. M., \& Martínez-Restrepo, O. A. (2014). Funciones Neurobiológicas y Neuropsicológicas afectadas en pacientes con Enfermedad de Alzheimer: Implicaciones y Consecuencias en la Calidad de Vida. Cuadernos de Neuropsicología, 8(2): 242-265. https://doi. org/107714/cnps/8.2.206.

Perrin, P. B. (2014). Racial/ethnic disparities in mental health over the first 2 years after traumatic brain injury: a model systems study. Archives of Physical Medicine and Rehabilitation, 95(12):2288-95. https://doi.org/ 10.1016/j.apmr.2014.07.409.

Richards, C. L., Malouin, F., \& Nadeau, S. (2015). Stroke rehabilitation: clinical picture, assessment, and therapeutic challenge. Progress in Brain Research, 218:253-80. https://doi.org/ 10.1016/ bs.pbr.2015.01.003.

Sadighi, A., Groody, A., Wasko, L., Hornak, J., \& Zand, R. (2018). Recognition of Stroke Warning Signs and Risk Factors Among Rural Population in Central Pennsylvania. Journal of Vascular and Interventional Neurology, 10(2):4-10.

Starosta, M., Kostka, J., \& Miller, E. (2018). Force analysis of shoulder joint muscles in the early phase of brain stroke. Acta Bioengineering and Biomechanics, 20(4):107-113.

Strizzi J., \& Cols. (2015). Sexual Functioning, Desire, and Satisfaction in Women with TBI and Healthy Controls. Behavioural Neurology, 24:74-79. https:// doi.org/10.1155/2015/247479.

Yu, A. Y. X., Kapral, M. K., Fang, J., \& Redelmeier, D. A.
ISSN: 2711-3760
https://doi.org/10.47185/27113760.v1n1.15

(2018). Increased Risk of Traffic Injury After a Cerebrovascular Event. Stroke, 49(12):3006-3011. https://doi.org/10.1161/STROKEAHA.118.022657. 